\title{
Three-axis Modification of Coordinates Enables Accurate Stereotactic Targeting in Non-human Primate Brains of Different Sizes
}

\author{
Hyung-Sun Kim', Goo-Hwa Kang ${ }^{1}$, Hanlim Song ${ }^{2}$, Ra Gyung Kim², Ji-Young Park², \\ Jeong Ho Hwang ${ }^{1}$ and Hyoung-Ihl Kim ${ }^{2,3 *}$ \\ ${ }^{1}$ Animal model research group, Korea Institute of Toxicology, Jeongup 53212, ${ }^{2}$ Neuromodulation Lab, Department of \\ Biomedical Science and Engineering, Gwangju Institute of Science and technology, Gwangju 61005, ${ }^{3}$ Department of \\ Neurosurgery, Presbyterian Medical Center, Jeonju 54987, Korea
}

The brain grows with age in non-human primates (NHPs). Therefore, atlas-based stereotactic coordinates cannot be used directly to target subcortical structures if the size of the animal's brain differs from that used in the stereotactic atlas. Furthermore, growth is non-uniform across different cortical regions, making it difficult to simply apply a single brain-expansion ratio. We determined the skull reference lines that best reflect changes in brain size along the $X, Y$, and $Z$ axes and plotted the changes in reference-line length against the changes in body weight. The skull reference lines had a linear relationship with body weight. However, comparison of skull reference lines with body weight confirmed the non-uniform skull growth during postnatal development, with skull growth more prominent in the $X$ and $Y$ axes than the $Z$ axis. Comparing the differences between the atlas-based lengths and those calculated empirically from plot-based linear fits, we created craniometric indices that can be used to modify stereotactic coordinates along all axes. We verified the accuracy of the corrected stereotactic targeting by infusing dye into internal capsule in euthanized and preserved NHP brains. Our axis-specific, craniometric-index-adjusted stereotactic targeting enabled us to correct for targeting errors arising from differences in brain size. Histological verification showed that the method was accurate to within $1 \mathrm{~mm}$. Craniometric index-adjusted targeting is a simple and relatively accurate method that can be used for NHP stereotactic surgery in the general laboratory, without the need for high-resolution imaging.

Key words: Stereotaxy, Nonhuman primate, Skull, Body weight

\section{INTRODUCTION}

Experimental research in non-human primates (NHPs) is im-

Received January 15, 2019, Revised May 8, 2019,

Accepted May 13,2019

* To whom correspondence should be addressed. TEL: 82-62-715-3234, FAX: 82-62-715-5309 e-mail:hyoungihl@gist.ac.kr portant not only for validating the concepts derived from smallanimal experiments but also as a step toward possible human applications. A variety of experimental procedures are performed in NHPs that require accurate neuroanatomical targeting, including electrode placement, delivery of genes or drugs to the brain, and selective destruction of particular regions within the brain. Stereotactic techniques and instruments are commonly used to achieve such targeting in NHPs.

Stereotactic procedures require an accurate stereotactic atlas, 
illustrating anatomical structures in $X, Y$, and $Z$ coordinates with reference to standard points or lines. There are currently several NHP stereotactic atlases available; these atlases are based on highresolution MRI and precision histological slicing of the primate brain [1-3]. In most NHP atlases, the 2-D planes are arranged relative to the ear-bar zero plane (EBZ) passing through the interaural line and the infraorbital ridge; hence stereotactic coordinates are typically defined as the distance from cross point between EBZ and coronal interaural point orthogonal to EBZ along the $X, Y$, and $Z$ axes [1]. Nonetheless, it is often not possible to apply these stereotactic atlases to NHPs because the atlases are specific to NHPs of a certain body weight or brain size $[1,3]$. It is well known the NHP brain undergoes considerable changes in shape and weight after birth. For example, the brain of an infant rhesus macaque is approximately $68 \%$ the size of the adult brain [4]. The growth rate of the brain is relatively rapid in animals with a body weight below $5 \mathrm{~kg}$; however, there is only a very gradual increase in brain weight thereafter [5]. There is also variability in brain size with gender and at the individual level, even in adult monkeys [6]. Therefore, perfect alignment of EBZ to the stereotactic atlas will not always guarantee accurate targeting.

To overcome this lack of reliability, ventriculography and magnetic resonance imaging (MRI) can be used to identify encephalic landmarks such as the anterior commissure-posterior commissure line (AC-PC line) [6, 7]. Although this approach enables the correct stereotactic coordinates to be obtained with satisfactory accuracy, these techniques are not readily available to most researchers because they are complex and require costly facilities. Because these techniques exceed the capabilities of most laboratories, NHP research has generally been restricted to highly specialized laboratories. An alternative approach is to use skull landmarks derived from $\mathrm{x}$-rays, on the assumption that the size of skull and the size of the brain correlate with each other with respect to body size and gender $[4,8-10]$. However, the brain and the skull do not grow symmetrically and a simple allometric calculation from body weight and skull landmarks often fails to reflect the proper growth of the brain. There is disproportionate growth of the brain and skull across the different cortical regions: the postnatal change in volume is greatest in the frontal and temporal lobes and smallest in the occipital and parietal lobes. This indicates that there is greater anterior-posterior expansion of the brain in the frontal lobe and that the inter-aural line, which lies in the EBZ plane, moves caudally as the temporal lobe grows $[9,11]$.

To address these limitations, we performed computerized tomography (CT) and made craniometric measurements of the length of the skull reference lines and assessed how these vary with body weight. We evaluated the relative ability of the various skull reference lines to reflect the non-uniform growth of the brain along the $X, Y$, and $Z$ axes, and used this information to calculate craniometric indices to enable independent modification of the stereotactic coordinates along each axis. We injected dye into the internal capsule (a subcortical structure) using modified stereotactic coordinates and verified the results histologically. We believe that the three-axis modification of stereotactic coordinates described here will improve the accuracy of stereotactic targeting in NHPs, especially for researchers with limited access to specialized facilities.

\section{MATERIALS AND METHODS}

\section{Animals}

All animal care and handling was in accordance with the guidelines of the Association for the Assessment and Accreditation of Laboratory Animal Care (AAALAC). Animal experiments were approved by the Institutional Animal Care and Use Committee (IACUC) of the Korean Institute of Toxicology (KIT-1609-0339). A total of 34 male cynomolgus monkeys (Macaca fascicularis) of body weight $2.4 \mathrm{~kg}$ to $5.5 \mathrm{~kg}$ were used in this study. To reduce the chance of mismatches between body weight and brain size, we excluded NHPs whose body weight exceeded $5.5 \mathrm{~kg}$. All CT scans were performed under anesthesia (intramuscular injection of 5 $\mathrm{mg} / \mathrm{kg}$ ketamine) and all efforts were made to minimize animal suffering.

\section{Computerized scanning and data acquisition}

CT scans were performed with a 16-channel spiral CT scanner (Somatom Emotion, Siemens Healthcare, Munich, Germany). After induction of general anesthesia with an intramuscular injection of ketamine $(5 \mathrm{mg} / \mathrm{kg}$ ), the animal was placed in a prone position, the gantry was aligned with the orbitomeatal line, and the scan was performed with slice thickness of $0.6 \mathrm{~mm}$. After the scan, images were reconstructed into multiplanar reconstruction (MPR) views that visualizes the brain in axial, sagittal, and coronal slices via built-in software. The raw images were exported into the Dicom viewer (RadiAnt DICOM Viewer, Medixant, Poznan, Poland) for further analysis.

\section{Measurement of body weight and endocranial volume}

To assess the allometric correlation between body weight and endocranial volume, we made longitudinal measurements of the body weight and endocranial volume of all animals. Body weight was measured regularly with a digital scale (Mattler Toledo KCC150, Manualslib, USA) from the age of 35 months up to before CT scanning. Only the animals which showed the normal 
growth pattern were entered in this study. To measure the endocranial volume in vivo, we exported the $\mathrm{CT}$ data from each animal into Image (NIH, Bethesda, MD, USA) and calculated the volume from the vertex to the outer margin of the foramen magnum. Briefly, all brain images were transformed into a stack' and the scale was set. In each CT slice, a region of interest (ROI) representing the endocranial content was drawn manually. For each slice, the ROI area was multiplied by the slice thickness, and the values for all of the slices were summed to produce the total volume of the brain compartment.

\section{Skull reference lines}

Fig. 1 shows the various skull reference lines used in this study. From the reconstructed 3-D CT images, cranial landmarks including the internal auditory meatus (IAM), porion (PO), anterior clinoid process (ACP), glabella (GL), opisthocranion (OPC), infraorbitale ridge (IOR), tuberculum sellae (TS), ear-bar zero (EBZ), dorsum sellae (DS), foramen magnum (FM), and the inner skull of the vertex bone were identified, then appropriate CT planes were reconstructed so that pairs of cranial landmarks appeared in the same plane. Fifteen connecting lines (skull reference lines)

\section{A. X-axis}
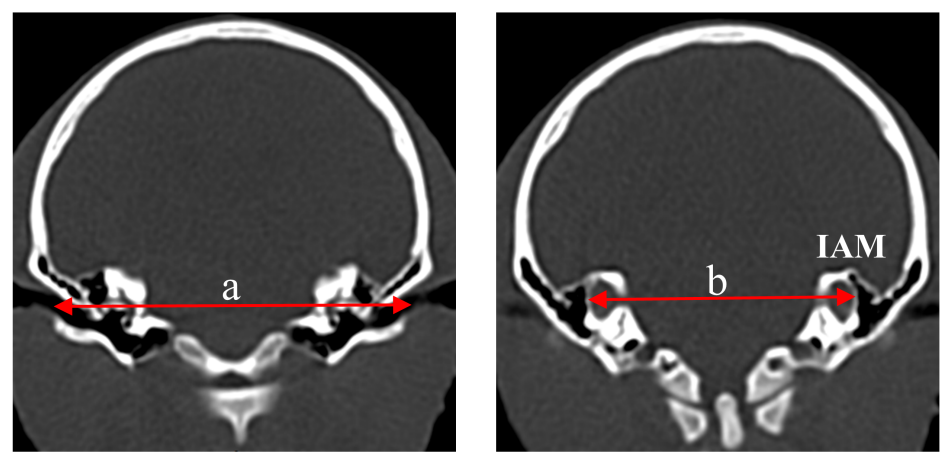

B. Y-axis
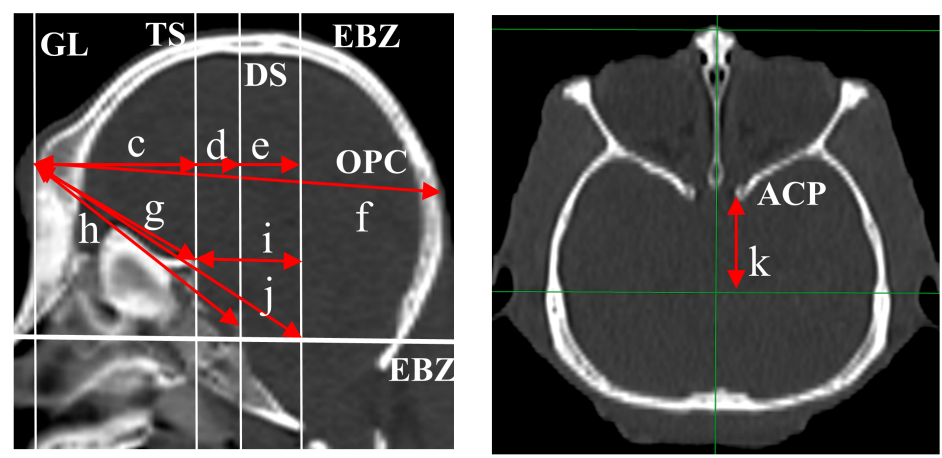

C. Z-axis

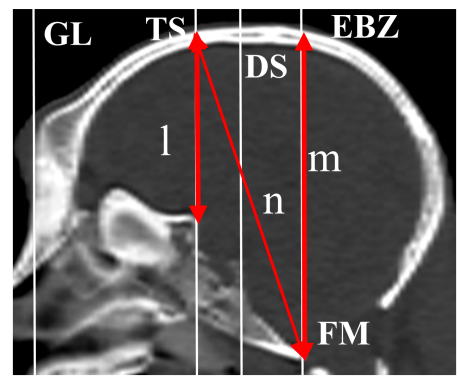

Fig. 1. Schematic drawing of skull reference lines (red) on CT planes. (A) Coronal slices showing the following $X$-axis reference lines: IPL (a) and IAL (b). (B) Sagittal slice showing the following $Y$-axis reference lines: GL-TS-VL (c), GL-DS-VL (d), GL-EBZ-VL (e), GL-OPC (f), GL-TS (g), GL-DS (h), TSEBZ-VL (i), and GL-EBZ (j). Horizontal slice showing the ACP-EBZ-VL reference line (k). (C) Sagittal slice showing the following $Z$-axis reference lines: TS-VVL (l), FM-VVL (m), and FM-TS-VVL (n). Together, these images illustrate the craniometric landmarks used in this study. IPL, internal porion line; IAM, internal auditory meatus; IAL, inter-auricular canal line; GL, glabella; VL, vertical line; TS, tuberculum sellae; DS, dorsum sellae; EBZ, ear-bar zero; OPC, opisthocranion; ACP, anterior clinoid process; IOR, infraorbital ridge; Po, porion; VVL, vertical vertex line; FM, foramen magnum. 
were drawn between pairs of cranial landmarks and their lengths measured with computer software (RadiAnt DICOM Viewer, Medixant, Poznan, Poland). These include the inter-auricular canal line (IAL) between the two internal auricular canals, the interporion line (IPL), the glabella- tuberculum sellae line (GL-TS), the glabella-tuberculum sellae vertical line (GL-TS-VL), the glabelladorsum sellae line (GL-DS), the glabella-dorsum sellae vertical line (GL-DS-VL), the glabella- opisthocranion line (GL-OPC), the infraorbital ridge-porion line (IOR-PO), the glabella-ear-bar zero line (GL-EBZ), the glabella-ear-bar zero vertical line (GL-EBZ$\mathrm{VL}$ ), the tuberculum sellae-ear-bar zero vertical line (TS-EBZ-VL), the anterior clinoid process-ear-bar zero vertical line (ACP-EBZVL), the tuberculum sellae-vertex vertical line (TS-VVL), the foramen magnum-vertex vertical line (FM-VVL), and the foramen magnum-tuberculum sellae-vertex vertical line (FM-TS-VVL). Among these lines, the IAL and IPL represent the variability of endocranial volume along the $\mathrm{X}$-axis (Fig. 1A) whereas GL-OPC, GL-EBZ, GL-EBZ-VL, GL-DS, GL-DS-VL, TS-EBZ-VL, GL-TS, GL-TS-VL, and ACP-EBZ-VL represent the variability along the $Y$-axis (Fig. 1B). TS-VVL, FM-VVL, and FM-TS-VVL represent the variability along the $Z$-axis (Fig. 1C). The values of these representative skull reference lines were compared with the animal's age, endocranial volume, and body weight to determine the most relevant linear plots.

\section{Modification of stereotactic coordinates via the craniomet- ric index}

Fig. 2 shows the process by which we calculated the craniometric indices, which we used to determine accurate stereotactic coordinates in animals whose body weight did not coincide with that of the animals used in the atlases. First, we plotted the length of the skull reference lines against body weight (Fig. 3) and fit the equation $y=\mathrm{K} x+\mathrm{B}$ to each plot, where $y$ is the length of the reference line, $x$ is body weight, $\mathrm{K}$ is the slope, and B is the $y$ intercept. We then selected the skull reference lines with the highest $R^{2}$ value, to enable the most reliable body-weight-dependent correction along each axis. Next, we used the equation parameters to predict accurate stereotactic coordinates for animals of a given body weight. For example, in determining X-coordinates in $2.5 \mathrm{~kg}$-weighted NHP, two distances (y) can be obtained from the equation of linear regression by inserting the body weights ( 2.5 and $4.9 \mathrm{~kg})$. After comparing the two distances, we can determine the "craniometric index" to reflect the ratio of different distance in X-axis skull reference line. The craniometric index is $>1$ if the body weight is bigger

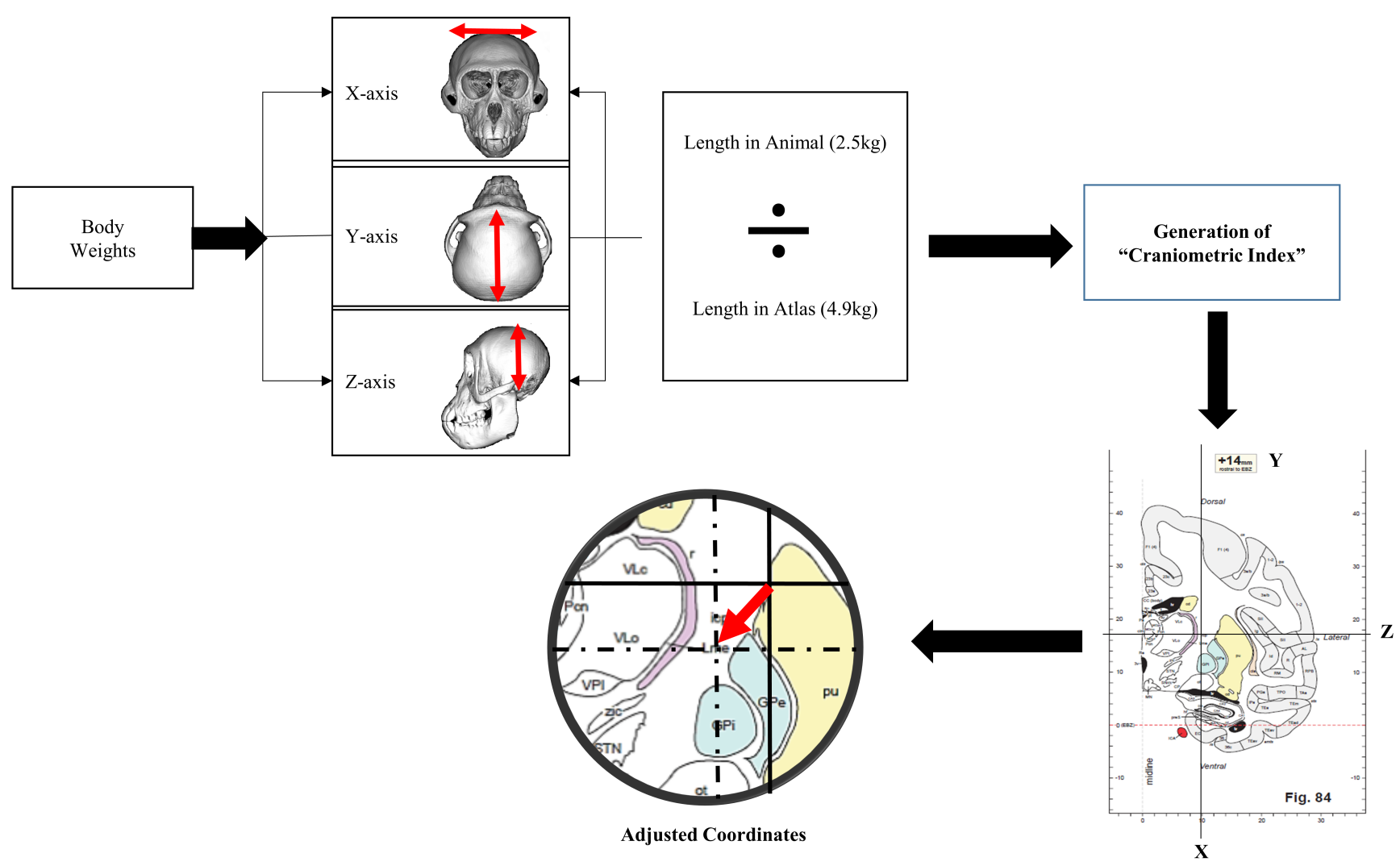

Fig. 2. Schematic showing the calculation of the craniometric index and the adjustment of stereotactic coordinates for the growing brain. 
than $4.9 \mathrm{~kg}$, and $<1$ if $4.9 \mathrm{~kg}$. The craniometric index is applied to $\mathrm{X}$-coordinate in $2.5 \mathrm{~kg}$-weighted NHP to determine the final Xcoordinates. This procedure is repeated separately for the $Y$-and $Z$-axis coordinates to compensate for the non-uniform changes in distance from EBZ with body weight. A similar equation is then applied to the $Y$ axis and the $Z$ axis but with different skull reference lines: GL-EBZ for the $Y$ axis and FM-VVL-TS for the $Z$ axis. The final, adjusted, $X, Y, Z$ coordinates can then be used to approach subcortical targets.

\section{Verification of target modification in animal carcasses}

Verification of targeting was performed in three cynomolgus monkeys which were previously euthanized and preserved in compliance with the institutional guidelines of the Korean Institute of Toxicology (KIT). Three animals with body weights of 2.4 $\mathrm{kg}, 3.0 \mathrm{~kg}$, and $4.1 \mathrm{~kg}$ were mounted in the stereotaxic apparatus.
Bilateral small trephination holes were made in the skull and, after EBZ zeroing along the $X$-, $Y$-, and $Z$ - axes, a microcannula (26 G) was lowered to the stereotactic target and $0.5 \mathrm{ml}$ of methylene blue was infused. The target site in the left hemisphere was derived directly from the stereotactic atlas, whereas the target site in the right hemisphere for corrected by applying the craniometric index to the coordinates. We aimed to target the posterior limb of the internal capsule, between the thalamus and globus pallidus $(X=+/-11$ $\mathrm{mm}, Y=13 \mathrm{~mm}, Z=18 \mathrm{~mm}$ from the EBZ point according to the atlas) $[1,13]$. After dye infusion, the brain was carefully removed from the skull cavity and sectioned to identify the dye stain. Two blind observers measured the distance between dye-staining point in the serially sectioned brain slices and target in stereotactic atlas, and then error distances were averaged in X, Y, Z axes. Two blind observers measured the distance between dye-staining point in the serially sectioned brain slices and target in stereotactic atlas,
A. IPL for $\mathrm{X}$-axis

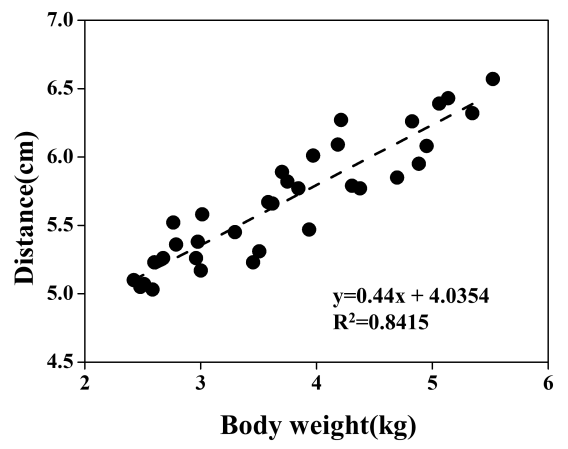

B. GL-EBZ for Y-axis

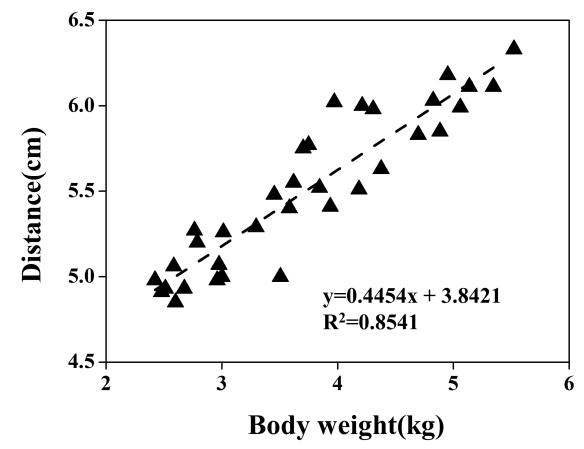

C. Mag-TS-VVL for Z-axis

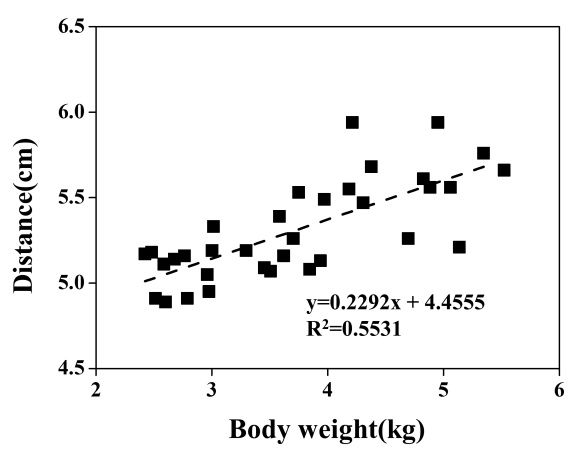

Fig. 3. Least squares regression plots of the relationships between the best-fitting skull reference lines and body weight. (A) IPL for X-axis has the highest R-squared value (0.8415). (B) GL-EBZ for Y-axis has the highest R-squared value (0.8541). (C) Mag-TS-VVL for Z-axis has the highest R-squared value $(0.5531)$.

A. Body Weight - Age

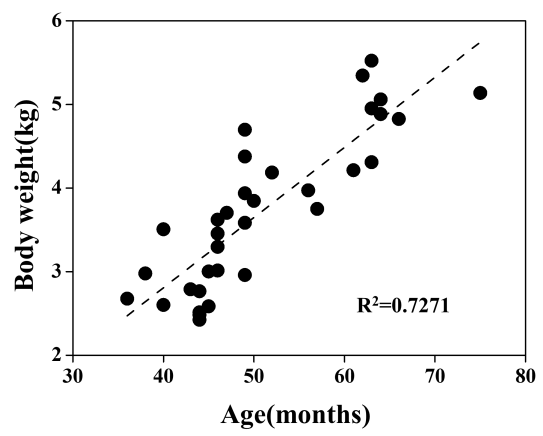

B. Body Weight - Endocranial Volume

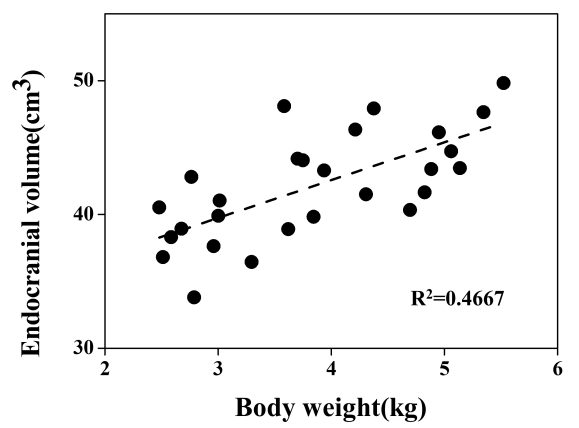

C. Age - Endocranial Volume

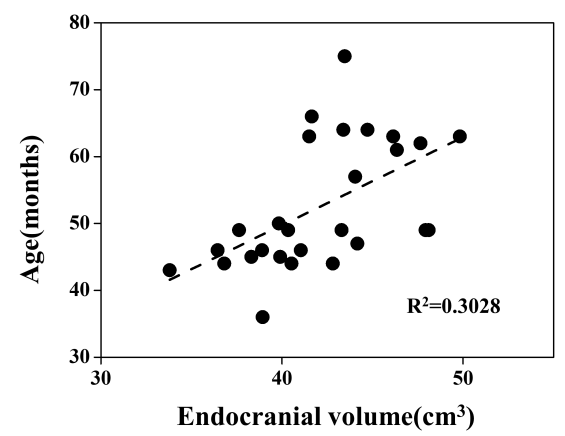

Fig. 4. Least squares regression plots for the relationships between body weight, age, and endocranial volume. (A) Simple regression between body weight and age. The $\mathrm{R}^{2}$ value of 0.7271 indicates that age accounts for $73 \%$ of the variability in body weight. (B) Simple regression between body weight and endocranial volume. The $\mathrm{R}^{2}$ value of 0.4667 indicates that body weight accounts for $47 \%$ of the variability in endocranial volume. (C) Simple regression between age and endocranial volume. The $\mathrm{R}^{2}$ value of 0.3028 indicates that age accounts for $30 \%$ of the variability in endocranial volume. 
Table 1. Raw data from the 34 non-human primates used in this study

\begin{tabular}{|c|c|c|c|c|c|c|c|c|c|c|c|c|c|c|c|c|c|c|c|}
\hline & $\begin{array}{l}\text { BW } \\
\text { (kg) }\end{array}$ & $\begin{array}{c}\text { Age } \\
\text { (mon- } \\
\text { th) }\end{array}$ & $\begin{array}{c}\text { Vol- } \\
\text { ume } \\
\text { (cm3) }\end{array}$ & IAL & $\underline{\text { IPL }}$ & $\begin{array}{c}\text { IOR- } \\
\text { PO }\end{array}$ & $\begin{array}{l}\text { GL- } \\
\text { OPC }\end{array}$ & $\begin{array}{l}\text { TS- } \\
\text { EBZ }\end{array}$ & $\begin{array}{l}\text { ACP- } \\
\text { EBZ } \\
\text { (Wag- } \\
\text { man's } \\
\text { land- } \\
\text { mark) }\end{array}$ & $\begin{array}{l}\underline{\text { GL- }} \\
\underline{\text { EBZ }} \\
\underline{\text { line }}\end{array}$ & $\begin{array}{l}\text { GL- } \\
\text { DS } \\
\text { line }\end{array}$ & $\begin{array}{l}\text { GL- } \\
\text { TS } \\
\text { line }\end{array}$ & $\begin{array}{c}\text { GL- } \\
\text { EBZ } \\
\text { point }\end{array}$ & $\begin{array}{c}\text { GL- } \\
\text { DS } \\
\text { point }\end{array}$ & $\begin{array}{c}\text { GL- } \\
\text { TS } \\
\text { point }\end{array}$ & $\begin{array}{l}\text { ACP- } \\
\text { VVL }\end{array}$ & $\begin{array}{l}\text { TS- } \\
\text { VVL }\end{array}$ & $\begin{array}{l}\text { FM- } \\
\text { VVL }\end{array}$ & $\begin{array}{l}\frac{\text { FM- }}{\text { TS- }} \\
\underline{\text { VVL }}\end{array}$ \\
\hline & & & & $\mathrm{X}$ & $\underline{X}$ & $\mathrm{Y}$ & $\mathrm{Y}$ & $\mathrm{Y}$ & $\mathrm{Y}$ & $\underline{\mathbf{Y}}$ & $\mathrm{Y}$ & $\mathrm{Y}$ & $\mathrm{Y}$ & $\mathrm{Y}$ & $\mathrm{Y}$ & Z & Z & Z & $\underline{\mathbf{Z}}$ \\
\hline 1 & 2.424 & 44 & & & 5.1 & 4.55 & 7.66 & 1.86 & 1.75 & 4.98 & 4 & 3.26 & 5.47 & 4.42 & 3.39 & 3.05 & 2.96 & 4.67 & 5.17 \\
\hline 2 & 2.481 & 44 & 40.53 & 3.69 & 5.05 & 4.53 & 7.62 & 1.96 & 1.78 & 4.91 & 3.78 & 3.03 & 5.42 & 4.34 & 3.2 & 2.95 & 2.77 & 4.75 & 5.18 \\
\hline 3 & 2.513 & 44 & 36.81 & 3.62 & 5.07 & 4.65 & 7.53 & 1.93 & 1.72 & 4.93 & 3.77 & 3.03 & 5.43 & 4.39 & 3.28 & 2.85 & 2.72 & 4.67 & 4.91 \\
\hline 4 & 2.585 & 45 & 38.31 & 3.75 & 5.03 & 4.79 & 7.63 & 1.98 & 1.91 & 5.06 & 3.94 & 3.12 & 5.64 & 4.43 & 3.35 & 2.88 & 2.82 & 4.85 & 5.11 \\
\hline 5 & 2.601 & 40 & & & 5.23 & 4.66 & 8.03 & 1.73 & 1.56 & 4.85 & 3.92 & 3.24 & 5.35 & 4.38 & 3.33 & 2.94 & 2.72 & 4.7 & 4.89 \\
\hline 6 & 2.676 & 36 & 38.93 & 3.68 & 5.26 & 4.72 & 7.7 & 1.77 & 1.66 & 4.93 & 3.95 & 3.22 & 5.45 & 4.48 & 3.33 & 2.85 & 2.72 & 4.88 & 5.14 \\
\hline 7 & 2.765 & 44 & 42.81 & 3.75 & 5.52 & 5.01 & 7.97 & 2.22 & 2.08 & 5.27 & 3.96 & 3.18 & 5.82 & 4.54 & 3.35 & 3.03 & 2.88 & 4.85 & 5.16 \\
\hline 8 & 2.788 & 43 & 33.80 & 3.79 & 5.36 & 4.86 & 8.04 & 2.05 & 1.82 & 5.2 & 4.04 & 3.18 & 5.68 & 4.63 & 3.4 & 2.76 & 2.6 & 4.53 & 4.91 \\
\hline 9 & 2.96 & 49 & 37.63 & 3.67 & 5.26 & 4.65 & 7.79 & 1.66 & 1.52 & 4.98 & 3.76 & 3.22 & 5.31 & 4.15 & 3.37 & 3.02 & 2.91 & 4.89 & 5.05 \\
\hline 10 & 2.977 & 38 & & & 5.38 & 4.99 & 7.87 & 2.15 & 1.97 & 5.07 & 3.87 & 3.09 & 5.65 & 4.43 & 3.24 & 2.73 & 2.67 & 4.49 & 4.95 \\
\hline 11 & 3.002 & 45 & 39.90 & 3.53 & 5.17 & 4.89 & 7.72 & 2.13 & 1.95 & 5 & 3.83 & 2.97 & 5.54 & 4.34 & 3.15 & 2.84 & 2.78 & 4.69 & 5.19 \\
\hline 12 & 3.014 & 46 & 41.04 & 3.72 & 5.58 & 5.14 & 7.8 & 2.07 & 1.94 & 5.26 & 3.95 & 3.26 & 5.74 & 4.64 & 3.46 & 3.18 & 2.99 & 4.82 & 5.33 \\
\hline 13 & 3.296 & 46 & 36.44 & 3.42 & 5.45 & 5.06 & 8.16 & 2.38 & 2.18 & 5.29 & 3.91 & 3.03 & 5.84 & 4.52 & 3.25 & 2.78 & 2.59 & 4.78 & 5.19 \\
\hline 14 & 3.454 & 46 & & 3.73 & 5.23 & 5.03 & 8.2 & 2.27 & 2.07 & 5.48 & 4.19 & 3.26 & 5.91 & 4.63 & 3.41 & 2.67 & 2.55 & 4.55 & 5.09 \\
\hline 15 & 3.506 & 40 & & & 5.31 & 4.59 & 7.68 & 1.87 & 1.78 & 5 & 3.76 & 3 & 5.32 & 4.19 & 3.1 & 2.84 & 2.66 & 4.76 & 5.07 \\
\hline 16 & 3.584 & 49 & 48.10 & 3.88 & 5.67 & 5.37 & 8.16 & 2.34 & 2.17 & 5.4 & 4.03 & 3.2 & 5.92 & 4.71 & 3.44 & 3.15 & 2.97 & 4.88 & 5.39 \\
\hline 17 & 3.62 & 46 & 38.90 & 3.86 & 5.66 & 5.19 & 8.16 & 2.16 & 1.99 & 5.55 & 4.28 & 3.47 & 6.01 & 4.81 & 3.62 & 2.78 & 2.71 & 4.6 & 5.16 \\
\hline 18 & 3.703 & 47 & 44.17 & 3.73 & 5.89 & 5.37 & 8.52 & 2.34 & 2.19 & 5.75 & 4.49 & 3.55 & 6.13 & 4.84 & 3.67 & 3.04 & 2.93 & 4.96 & 5.26 \\
\hline 19 & 3.749 & 57 & 44.04 & 4.08 & 5.82 & 5.4 & 8.47 & 2.43 & 2.21 & 5.77 & 4.3 & 3.37 & 6.12 & 4.76 & 3.46 & 2.9 & 2.69 & 4.93 & 5.53 \\
\hline 20 & 3.845 & 50 & 39.82 & 3.88 & 5.77 & 5 & 8.23 & 2.25 & 2.05 & 5.52 & 4.2 & 3.33 & 6.05 & 4.66 & 3.51 & 2.99 & 2.82 & 4.6 & 5.08 \\
\hline 21 & 3.937 & 49 & 43.29 & 3.76 & 5.47 & 5.33 & 8.56 & 2.15 & 2.03 & 5.41 & 4.24 & 3.34 & 5.96 & 4.68 & 3.58 & 2.93 & 2.87 & 4.85 & 5.13 \\
\hline 22 & 3.972 & 56 & & & 6.01 & 5.47 & 8.73 & 2.39 & 2.18 & 6.02 & 4.74 & 3.55 & 6.39 & 5.16 & 3.72 & 2.98 & 2.82 & 4.89 & 5.49 \\
\hline 23 & 4.185 & 52 & & & 6.09 & 5.23 & 8.08 & 2.32 & 2.09 & 5.51 & 4.09 & 3.22 & 6.04 & 4.49 & 3.45 & 3.01 & 3 & 5.14 & 5.55 \\
\hline 24 & 4.212 & 61 & 46.35 & 3.78 & 6.27 & 5.67 & 8.53 & 2.47 & 2.25 & 6 & 4.57 & 3.8 & 6.55 & 5.2 & 3.95 & 3.43 & 3.24 & 5.27 & 5.94 \\
\hline 25 & 4.308 & 63 & 41.50 & 3.77 & 5.79 & 5.51 & 8.54 & 2.47 & 2.31 & 5.98 & 4.66 & 3.68 & 6.6 & 5.07 & 3.87 & 3.07 & 2.91 & 4.82 & 5.47 \\
\hline 27 & 4.376 & 49 & 47.93 & 3.95 & 5.77 & 5.36 & 8.36 & 2.29 & 2.17 & 5.63 & 4.23 & 3.16 & 6.03 & 4.66 & 3.36 & 3.06 & 2.96 & 5.16 & 5.68 \\
\hline 26 & 4.696 & 49 & 40.34 & 3.75 & 5.85 & 5.43 & 8.24 & 2.38 & 2.15 & 5.83 & 4.53 & 3.59 & 6.22 & 4.92 & 3.72 & 2.9 & 2.72 & 4.8 & 5.26 \\
\hline 28 & 4.825 & 66 & 41.65 & 3.97 & 6.26 & 5.91 & 8.8 & 2.73 & 2.53 & 6.03 & 4.45 & 3.42 & 6.46 & 4.86 & 3.54 & 2.94 & 2.77 & 4.68 & 5.61 \\
\hline 29 & 4.885 & 64 & 43.39 & 3.63 & 5.95 & 5.47 & 8.29 & 2.32 & 2.13 & 5.85 & 4.58 & 3.48 & 6.27 & 5.09 & 3.67 & 3.25 & 3.09 & 4.84 & 5.56 \\
\hline 30 & 4.952 & 63 & 46.14 & 3.59 & 6.08 & 5.79 & 8.67 & 2.46 & 2.12 & 6.18 & 4.76 & 3.81 & 6.67 & 5.41 & 4 & 3.51 & 3.27 & 5.45 & 5.94 \\
\hline 31 & 5.06 & 64 & 44.72 & 3.85 & 6.39 & 5.56 & 8.8 & 2.42 & 2.2 & 5.99 & 4.65 & 3.57 & 6.45 & 5.05 & 3.78 & 3 & 2.89 & 5.06 & 5.56 \\
\hline 32 & 5.138 & 75 & 43.46 & 4.02 & 6.43 & 5.79 & 8.76 & 2.51 & 2.23 & 6.11 & 4.61 & 3.69 & 6.54 & 4.99 & 3.77 & 3 & 2.77 & 4.78 & 5.21 \\
\hline 33 & 5.346 & 62 & 47.65 & 4.02 & 6.32 & 5.79 & 8.66 & 2.52 & 2.4 & 6.11 & 4.83 & 3.61 & 6.61 & 5.3 & 3.86 & 3.3 & 3.18 & 4.98 & 5.76 \\
\hline 34 & 5.523 & 63 & 49.83 & 4.12 & 6.57 & 5.94 & 8.92 & 2.57 & 2.43 & 6.33 & 5.01 & 3.81 & 6.81 & 5.5 & 4 & 3.2 & 3.04 & 4.85 & 5.66 \\
\hline $\begin{array}{l}\text { BW- } \\
\text { R2 }\end{array}$ & 1.0000 & 0.7271 & 0.4667 & 0.2757 & 0.8415 & 0.8269 & 0.7522 & 0.6643 & 0.6391 & 0.8541 & 0.7762 & 0.6052 & 0.8136 & 0.6915 & 0.6313 & 0.2648 & 0.2332 & 0.2116 & 0.5531 \\
\hline $\begin{array}{l}\mathrm{p} \text { - } \\
\text { value }\end{array}$ & & .000 & .000 & .004 & .000 & .000 & .000 & .000 & .000 & .000 & .000 & .000 & .000 & .000 & .000 & .002 & .004 & .006 & .000 \\
\hline $\begin{array}{l}\text { F-p- } \\
\text { value }\end{array}$ & & .000 & .000 & .004 & .000 & .000 & .000 & .000 & .000 & .000 & .000 & .000 & .000 & .000 & .000 & .002 & .004 & .006 & .000 \\
\hline $\begin{array}{l}\text { Age- } \\
\text { R2 }\end{array}$ & 0.7271 & 1.0000 & 0.3028 & 0.2333 & 0.7201 & 0.7255 & 0.6621 & 0.6008 & 0.5365 & 0.7695 & 0.6660 & 0.6054 & 0.7548 & 0.6144 & 0.6183 & 0.3276 & 0.2540 & 0.1850 & 0.5120 \\
\hline
\end{tabular}


Table 1. Continued

\begin{tabular}{|c|c|c|c|c|c|c|c|c|c|c|c|c|c|c|c|c|c|c|c|}
\hline & $\begin{array}{c}\text { BW } \\
\text { (kg) }\end{array}$ & $\begin{array}{c}\text { Age } \\
\text { (mon- } \\
\text { th) }\end{array}$ & $\begin{array}{c}\text { Vol- } \\
\text { ume } \\
(\mathrm{cm} 3)\end{array}$ & IAL & $\underline{\text { IPL }}$ & $\begin{array}{c}\text { IOR- } \\
\text { PO }\end{array}$ & $\begin{array}{l}\text { GL- } \\
\text { OPC }\end{array}$ & $\begin{array}{c}\text { TS- } \\
\text { EBZ }\end{array}$ & $\begin{array}{l}\text { ACP- } \\
\text { EBZ } \\
\text { (Wag- } \\
\text { man's } \\
\text { land- } \\
\text { mark) }\end{array}$ & $\begin{array}{l}\frac{\text { GL- }}{\text { EBZ }} \\
\underline{\text { line }}\end{array}$ & $\begin{array}{l}\text { GL- } \\
\text { DS } \\
\text { line }\end{array}$ & $\begin{array}{c}\text { GL- } \\
\text { TS } \\
\text { line }\end{array}$ & $\begin{array}{c}\text { GL- } \\
\text { EBZ } \\
\text { point }\end{array}$ & $\begin{array}{c}\text { GL- } \\
\text { DS } \\
\text { point }\end{array}$ & $\begin{array}{c}\text { GL- } \\
\text { TS } \\
\text { point }\end{array}$ & $\begin{array}{l}\text { ACP- } \\
\text { VVL }\end{array}$ & $\begin{array}{c}\text { TS- } \\
\text { VVL }\end{array}$ & $\begin{array}{l}\text { FM- } \\
\text { VVL }\end{array}$ & $\begin{array}{l}\frac{\text { FM- }}{\text { TS- }} \\
\underline{\text { VVL }}\end{array}$ \\
\hline & & & & X & $\underline{\mathbf{X}}$ & Y & Y & Y & $\mathrm{Y}$ & $\underline{\mathbf{Y}}$ & Y & Y & Y & Y & Y & Z & Z & Z & $\underline{\mathbf{Z}}$ \\
\hline $\begin{array}{l}\mathrm{P} \\
\text { value }\end{array}$ & .000 & & .003 & .009 & .000 & .000 & .000 & .000 & .000 & .000 & .000 & .000 & .000 & .000 & .000 & .000 & .002 & .011 & .000 \\
\hline $\begin{array}{l}\text { F-p- } \\
\text { value }\end{array}$ & .000 & & .003 & .009 & .000 & .000 & .000 & .000 & .000 & .000 & .000 & .000 & .000 & .000 & .000 & .000 & .002 & .011 & .000 \\
\hline $\begin{array}{l}\text { Vol. } \\
-\mathrm{R}^{2}\end{array}$ & 0.4667 & 0.3028 & 1.0000 & 0.2962 & 0.4917 & 0.5376 & 0.4362 & 0.3781 & 0.4312 & 0.4662 & 0.4387 & 0.3303 & 0.4601 & 0.4638 & 0.3563 & 0.5242 & 0.5337 & 0.4204 & 0.6277 \\
\hline $\begin{array}{l}\mathrm{p}- \\
\text { value }\end{array}$ & .000 & .003 & & .003 & .000 & .000 & .000 & .001 & .000 & .000 & .000 & .002 & .000 & .000 & .001 & .000 & .000 & .000 & .000 \\
\hline $\begin{array}{l}\text { F-p- } \\
\text { value }\end{array}$ & .000 & .003 & & .003 & .000 & .000 & .000 & .001 & .000 & .000 & .000 & .002 & .000 & .000 & .001 & .000 & .000 & .000 & .000 \\
\hline
\end{tabular}

then error distances were averaged in $\mathrm{X}, \mathrm{Y}, \mathrm{Z}$ axes.

\section{Statistical analysis}

To determine the statistical correlations between age, body weight, endocranial volume, and the skull reference lines, we performed a simple linear regression analysis in ORIGIN 8.5 software (OriginLab, Northampton, MA, USA). This yielded $\mathrm{R}^{2}$ values $(\mathrm{p}<0.001)$ and a simple regression equation for each variable such as body weight, age, and endocranial volume. We assessed all the correlations between the variables and the different skull reference lines and identified the lines that produced the greatest $\mathrm{R}^{2}$ value. An overall $F$ test $(\mathrm{p}<0.0001)$ was performed to evaluate the regression model. During validation of the results, we compared the errors (distance from the planned target) using Student's t-test $(\mathrm{p}<0.05)$.

\section{RESULTS}

\section{Relationships between body weight, age, and endocranial volume}

Fig. 4 shows the relationships between body weight, age and, endocranial volume for 34 cynomolgus NHPs. All measurements are presented in Table 1. There was an overall increase in body weight with age for body weights between $2.4 \mathrm{~kg}$ and $5.5 \mathrm{~kg}$ (overall $\mathrm{R}^{2}=0.73, \mathrm{p}<0.0001$ ). Beyond 60 months of age, there was no increase in body weight (Fig. 4A). Fig. 4B and $4 \mathrm{C}$ show the relationships between body weight or age and endocranial volume. The correlation score for the relationship between body weight and endocranial volume $\left(\mathrm{R}^{2}=0.47\right)$ was statistically higher than the correlation score for the relationship between age and endocranial volume $\left(\mathrm{R}^{2}=0.30\right)$.
Table 2. $\mathrm{R}^{2}$ values for the relationships between representative skull reference lines and body weight, age, and endocranial volume

\begin{tabular}{lccc}
\hline & $\begin{array}{c}\text { X-Axis }\left(\mathbf{R}^{2}\right) \\
\text { IPL }\end{array}$ & $\begin{array}{c}\text { Y-Axis }\left(\mathbf{R}^{2}\right) \\
\text { GL-EBZ }\end{array}$ & $\begin{array}{c}\text { Z-Axis }\left(\mathbf{R}^{2}\right) \\
\text { Mag-TS- } \\
\text { VVL }\end{array}$ \\
\hline BW & 0.8415 & 0.8541 & 0.5531 \\
Age & 0.7201 & 0.7695 & 0.5120 \\
Endocranial volume & 0.4917 & 0.4662 & 0.6277 \\
\hline
\end{tabular}

The length of skull reference lines changes with age, endocranial volume, and body weight

Table 2 and Fig. 3 show the relationships between skull reference lines representing the stereotactic $X, Y$, and $Z$ axes, and age, endocranial volume, and body weight. When the length of skull reference lines was compared with age, endocranial volume, and body weight, the relationship with body weight was the strongest $\left(\mathrm{R}^{2}=0.73\right)$. Among the skull reference lines, the IPL had the highest $\mathrm{R}^{2}$ value of all $X$-axis lines $\left(\mathrm{R}^{2}=0.84, \mathrm{p}<0.0001\right)$ and the GLEBZ line had the highest the $\mathrm{R}^{2}$ value of all $\mathrm{Y}$-axis lines $\left(\mathrm{R}^{2}=0.85\right.$, $\mathrm{p}<0.0001)$. Among the $Z$-axis reference lines, the Mag-TS-VVL line had the highest $R^{2}$ value $\left(R^{2}=0.55, p<0.0001\right)$. Our results confirm that skull growth is uneven during postnatal development and suggest that a simple brain expansion ratio would be unlikely to reflect the true growth. Skull growth was more prominent in the $X$ and $Y$ axes than the $Z$ axis; consequently, use of skull reference lines that correspond to the different axes is a reasonable approach to determine the individual $X, Y$, and $Z$ coordinates for the growing brain. Once a skull reference line was selected for each axis, body weight proved to be the most reliable determinant of the length of the reference line from EBZ. 


\section{Craniometric index}

The craniometric index was calculated as the ratio of the reference line length in the atlas to the reference line length derived from the simple linear regression equation. The craniometric equation was calculated separately for each axis, using the reference line with the highest $\mathrm{R}^{2}$ value for that axis. Table 3 shows the values used to calculate the craniometric indices for each axis for a $2.5 \mathrm{~kg}$ NHP. The craniometric index was similar for the $X$ and $Y$ axes, but larger for the $Z$ axis.

\section{Validation of the accuracy of modified target coordinates}

Modification of stereotactic targets by the axis-specific craniometric indices was validated by microinjection of methylene blue. Fig. 5 shows the dye staining after injections for multiple targets for the internal capsule, caudate \& putamen and substantia nigra in animals of different body weights. For example, in the NPH with a body weight of $2.4 \mathrm{~kg}$, stereotactic microinjection of methylene blue according to the atlas coordinates $(X: 11 ; Y: 13 ; Z: 18)$ stained the upper putamen, outside the internal capsule. We could achieve a similar results in targeting the caudate nucleus, putamen and substantia nigra in 2.4,3 or 4 kg-weighted NPH (Fig. 5).

Table 3. Example values used to calculate the craniometric indices in a $2.5 \mathrm{~kg}$ NHP

\begin{tabular}{clccccc}
\hline & & K & B & Atlas (4.9 kg) & $\begin{array}{c}\text { Experimental } \\
\text { animal (2.5 kg) }\end{array}$ & $\begin{array}{c}\text { Craniometric } \\
\text { index }\end{array}$ \\
\cline { 5 - 6 } & Scatter plot used & (slope of plot) & (y intercept) & Distance & Distance \\
X & BW/IPL plot & 0.44 & 4.0354 & 6.19 & 5.14 & 0.83 \\
Y & BW/GL-EBZ line plot & 0.4454 & 3.8421 & 6.02 & 4.96 \\
Z & BW/FM-TS-VVL plot & 0.2292 & 4.4555 & 5.58 & 5.03 \\
\hline
\end{tabular}

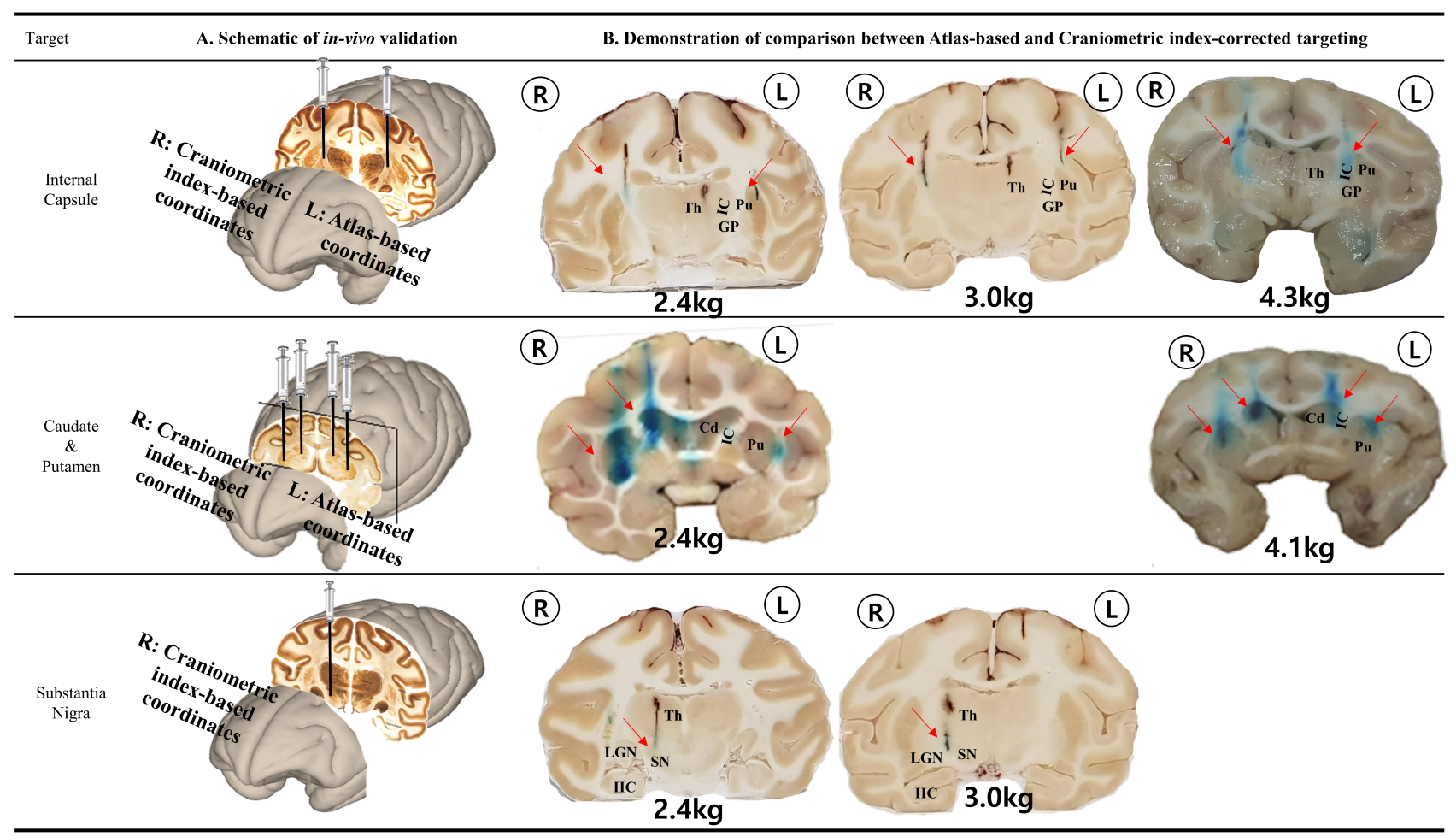

Fig. 5. In-vivo validation of the craniometric index-adjusted stereotactic coordinates for multiple targets in the brains of cynomolgus monkeys with different body weights. (A) Schematic drawings of dye injection for multiple targets. Injection into left hemisphere showed the atlas-based targets whereas injection into right hemisphere indicates the craniometric index-adjusted targets. (B) Different dye markers for multiple targets such as internal capsule, caudate \& putamen and substantia nigra between atlas-based and craniometric index-corrected targeting in $2.4 \mathrm{~kg}, 3.0 \mathrm{~kg}, 4.1 \mathrm{~kg}$ or $4.3 \mathrm{~kg}$ cynomolgus monkey. Cd, caudate nucleus; GP, globus Pallidus; HC, hippocampus; IC, internal capsule; LGN, lateral geniculate nucleus; Pu, putamen; SN, substantia nigra; Th, thalamus. 
Table 4. Errors in targeting the internal capsule with dye. In-vivo validation of the craniometric-index adjusted coordinates

\begin{tabular}{|c|c|c|c|c|c|c|c|c|c|c|c|c|c|}
\hline \multirow{3}{*}{$\begin{array}{l}\text { BW } \\
(\mathrm{kg})\end{array}$} & \multirow{3}{*}{$\begin{array}{l}\text { Tar- } \\
\text { get } \\
\text { Site }\end{array}$} & \multicolumn{6}{|c|}{ Coordinates in Atlas } & \multicolumn{6}{|c|}{ Coordinates in animal } \\
\hline & & \multicolumn{3}{|c|}{$\begin{array}{l}\text { Atlas-based } \\
\text { target }\end{array}$} & \multicolumn{3}{|c|}{$\begin{array}{c}\text { Error distance } \\
\text { (averaged) }\end{array}$} & \multicolumn{3}{|c|}{$\begin{array}{l}\text { Modified target by } \\
\text { craniometric index }\end{array}$} & \multicolumn{3}{|c|}{$\begin{array}{c}\text { Error distance } \\
\text { (averaged) }\end{array}$} \\
\hline & & $\mathbf{X}$ & $\mathbf{Y}$ & $\mathbf{Z}$ & $\mathbf{X}$ & $\mathbf{Y}$ & $\mathbf{Z}$ & $\mathbf{X}$ & $\mathbf{Y}$ & $\mathbf{Z}$ & $\mathbf{X}$ & $\mathbf{Y}$ & $\mathbf{Z}$ \\
\hline 2.4 & IC & 11 & 13 & 18 & $\begin{array}{l}13.33 \pm 0.47 \\
(23 \%)\end{array}$ & $\begin{array}{l}14.67 \pm 0.47 \\
(12 \%)\end{array}$ & $\begin{array}{c}19.33 \pm 0.47 \\
(6 \%)\end{array}$ & 9.02 & 10.66 & 16.2 & $\begin{array}{c}11.33 \pm 0.47 \\
(5 \%)\end{array}$ & $\begin{array}{c}12.5 \pm 0.0 \\
(-4 \%)^{*}\end{array}$ & $\begin{array}{r}20 \pm 0.0 \\
(11 \%)\end{array}$ \\
\hline 3.0 & IC & 11 & 13 & 18 & $\begin{array}{l}12.25 \pm 0.25 \\
\quad(11 \%)\end{array}$ & $\begin{array}{l}15.00 \pm 0.00 \\
(15 \%)\end{array}$ & $\begin{array}{c}18.5 \pm 0.5 \\
(3 \%)\end{array}$ & 9.46 & 11.18 & 16.56 & $\begin{array}{c}11.5 \pm 0.5 \\
(5 \%)\end{array}$ & $\begin{array}{l}12.75 \pm 0.25 \\
(-2 \%)^{* *}\end{array}$ & $\begin{array}{c}19 \pm 0.0 \\
(6 \%)\end{array}$ \\
\hline 4.3 & IC & 11 & 13 & 18 & $\begin{array}{c}12.5 \pm 0.5 \\
(14 \%)\end{array}$ & $\begin{array}{c}12.25 \pm 0.25 \\
(-6 \%)\end{array}$ & $\begin{array}{l}19 \pm 0 \\
(6 \%)\end{array}$ & 10.56 & 12.48 & 17.64 & $\begin{array}{c}12 \pm 1.0 \\
(9 \%)\end{array}$ & $\begin{array}{c}12.25 \pm 0.25 \\
(-6 \%)^{*}\end{array}$ & $\begin{array}{c}19 \pm 0.0 \\
(6 \%)\end{array}$ \\
\hline
\end{tabular}

${ }^{*} \mathrm{p}<0.05,{ }^{* *} \mathrm{p}<0.01$.

Table 4 shows a comparison of the stereotactic coordinates for the right internal capsule in a $2.5 \mathrm{~kg}$ NHP derived from the stereotactic atlas, together with the coordinates derived after craniometric index corrections. The $X, Y$, and $Z$ coordinates were predicted from linear graphs of body weight and each axis was represented by a different skull reference line.

The average errors were 23\% ( $X$ axis), 12\% ( $Y$ axis), and 6\% ( $Z$ axis) in a $2.4-k g$ NHP, $11 \%$ ( $X$ axis), $15 \%$ ( $Y$ axis), and $3 \%$ ( $Z$ axis) in a $3-\mathrm{kg}$ NHP, and $14 \%$ ( $X$ axis), $6 \%$ ( $Y$ axis), and $6 \%$ ( $Z$ axis) in a 4.3 $\mathrm{kg}$ NHP. However, after using the craniometric indices to adjust the stereotactic coordinates, the dye stained the upper portion of the internal capsule correctly in all specimens. The average errors were 5\% ( $X$ axis), $4 \%$ ( $Y$ axis), and 11\% ( $Z$ axis) in the 2.4-kg NHP, $5 \%$ ( $X$ axis), $2 \%$ ( $Y$ axis), and 6\% ( $Z$ axis) in the $3-\mathrm{kg}$ NHP, and $9 \%(X$ axis), $6 \%$ ( $Y$ axis), and $6 \%$ ( $Z$ axis) in the $4.3-\mathrm{kg}$ NHP.

\section{DISCUSSION}

In this study, we identified reliable skull reference lines that can be used to adjust stereotactic coordinates to take into account disproportionate skull growth along the different axes. Among the skull reference lines, the inter-porion line (IPL) and the glabellaear-bar zero (GL-EBZ) line had the highest $\mathrm{R}^{2}$ values for the $X$ $\left(\mathrm{R}^{2}=0.84\right)$ and $Y\left(\mathrm{R}^{2}=0.85\right)$ axes, respectively. We also compared the length of the skull reference lines with body-weight variability in 34 male cynomolgus monkeys. After deriving linear fits to the scatter plots of body weight and reference line length, we calculated craniometric indices that can be used to adjust the stereotactic coordinates for a particular target along each axis. The adjusted target coordinates proved to be accurate for subcortical targeting, despite the use of simple stereotactic apparatus and the lack of high-resolution MRI imaging. These results will therefore facilitate NHP experiments in cynomolgus monkeys (Macaca fascicularis) in the general laboratory setting. The use of craniometric indices can enhance the accuracy of subcortical targeting by accounting for differences in brain size, leading the wider application of stereotactic procedures in NHPs.

Stereotactic surgery in NHPs is complex and usually requires access to a costly imaging facility to accurately identify the intracerebral target. We previously designed a simplified adaptor that can be mounted on a small-animal stereotactic frame and that enables easy atlas-based stereotactic targeting of subcortical structures in NHPs without the need for high-resolution imaging [13]. However, brain size varies with sex, age, body weight, and environmental factors such as obesity, disease, etc. Because the standard stereotactic atlases are based on a brain of fixed weight, they do not provide information on how to adjust the coordinates to account for variability in brain size and weight [1]. Although this problem can be overcome with high-resolution MRI imaging, a fiducialmarker-equipped, expensive stereotactic frame, and computeraided targeting, most general laboratories do not have access to such facilities.

The stereotactic atlas provides the distances from EBZ in a NHP with fixed body weight $[1,2]$. When we compared the endocranial volume and skull growth with age and body weight, body weight had a stronger relationship with overall brain expansion than age. However, the brain grows disproportionately along the different axes, rotating in forward and downward directions as body weight increases [5]. Consequently, the use of a simple magnification ratio to account for brain expansion does not conform to the characteristics of patterns in brain growth [11]. We selected the skull reference lines that reflect the changes of brain size separately for the $X, Y$ and $Z$ axes. Among the skull reference lines for the $X$ axis, the IPL showed the highest $\mathrm{R}^{2}$ value $\left(\mathrm{R}^{2}=0.84, \mathrm{p}<0.0001\right)$; for the $Y$ axis, the GL-EBZ line showed the highest the $R^{2}$ value $\left(R^{2}=0.85\right.$, $\mathrm{p}<0.0001)$. For the $Z$ axis, the Mag-TS-VVL line showed the highest $R^{2}$ value $\left(R^{2}=0.55, p<0.0001\right)$. Of all the reference lines assessed, the length of these lines most directly reflected the non-uniform 
growth of the skull with increases in body weight.

Historically, the determination of stereotactic coordinates was based on skull reference lines $[11,14,15]$. However, this led to controversial results in the stereotactic targeting, and ultimately to recommendation to use the anterior commissure - posterior commissure (AC-PC) line instead [6]. Nonetheless, Wagman et al. recommend using the oblique anterior clinoid process - EBZ distance to modify the coordinates for the $X$ and $Y$ axes and the distance between the base of pituitary fossa and the horizontal plane (H plane) to modify the $Z$ axis coordinates. However, the correction for the $\mathrm{X}$ axis was not clear [5]. Aggleton et al. recommend using the posterior tip of the sphenoid bone for stereotactic targeting of the amygdala, hypothalamus, and basal forebrain. However, no previous studies have proposed a universal method for modifying all three axes during the targeting of subcortical regions. Our study is the first to propose a method for separate correction along the $X, Y$, and $Z$ axes, to account for the disproportionate skull growth in the different directions. Oblique line-based corrections may distort the distance along a single axis; therefore separate, proportional correction via the craniometric indices is critical to reduce targeting errors.

This study has some limitations. First, the craniometric index corrections do not provide sub-millimeter precision. Even after applying this technique, some errors may exist. Therefore, this technique is not recommended for stereotactic targeting requiring sub-millimeter accuracy. Second, the relationship between body weight and the length of the skull reference lines is not universal. We attempted to minimize errors by excluding body weights that fell outside of the normal growth pattern and by limiting the range of body weights in the study to $2.4 \sim 5.5 \mathrm{~kg}$. Within these constraints, we found a relatively strong relationship between body weight and the length of the skull reference lines. We believe that staying within these constraints is important for the accuracy of the craniometric-index modifications.

In summary, we described skull reference lines that reflect the changes in brain size with changes in body weight. Craniometric indices based on reference-line measurements can be used to adjust atlas-based coordinates so that they are appropriate for a different brain size. Combined with our previously described simple stereotactic frame adaptor, the craniometric indices enable simple, inexpensive, and accurate targeting of subcortical structures.

\section{ACKNOWLEDGEMENTS}

This work was supported by Basic Science Research Program through National Research Foundation of Korea funded by the Ministry of Science and ICT (NRF-2016R1A2B3009660 \& NRF-
2019M3C1B8090841).

\section{REFERENCES}

1. Saleem K, Logothetis N (2016) A combined MRI and histology atlas of the rhesus monkey brain in stereotaxic coordinates. 2nd ed. Academic Press, Cambridge, MA.

2. Szabo J, Cowan WM (1984) A stereotactic atlas of the brain of the cynomolgus monkey. J Comp Neurol 222:265-300.

3. Martin RF, Bowden DM (1996) A stereotaxic template atlas of the macaque brain for digital imaging and quantitative neuroanatomy. Neuroimage 4:119-150.

4. Holt A, Cheek D, Mellits D, Hill D (1975) Fetal and postnatal cellular growth: hormones and nutrition. Wiley, New York, NY.

5. Wagman IH, Loeffler JR, McMillan JA (1975) Relationship between growth of brain and skull of Macaca mulatta and its importance for the stereotaxic technique. Brain Behav Evol 12:116-134.

6. Percheron G, Yelnik J, François C (1986) Systems of coordinates for stereotactic surgery and cerebral cartography: advantages of ventricular systems in monkeys. J Neurosci Methods 17:69-88.

7. Min HK, Ross EK, Lee KH, Dennis K, Han SR, Jeong JH, Marsh MP, Striemer B, Felmlee JP, Lujan JL, Goerss S, Duffy PS, Blaha C, Chang SY, Bennet KE (2014) Subthalamic nucleus deep brain stimulation induces motor network BOLD activation: use of a high precision MRI guided stereotactic system for nonhuman primates. Brain Stimulat 7:603-607.

8. Bronson R (1981) Brain weight-boidy weight relationship in 12 species of nonhuman primate. Am J Phys Anthropol 56:77-81.

9. Scott JA, Grayson D, Fletcher E, Lee A, Bauman MD, Schumann CM, Buonocore MH, Amaral DG (2016) Longitudinal analysis of the developing rhesus monkey brain using magnetic resonance imaging: birth to adulthood. Brain Struct Funct 221:2847-2871.

10. Isler K, Christopher Kirk E, Miller JM, Albrecht GA, Gelvin BR, Martin RD (2008) Endocranial volumes of primate species: scaling analyses using a comprehensive and reliable data set. J Hum Evol 55:967-978.

11. Aggleton JP, Passingham RE (1981) Stereotaxic surgery under $\mathrm{X}$-ray guidance in the rhesus monkey, with special reference to the amygdala. Exp Brain Res 44:271-276.

12. Choi K, Chang J, Lee MJ, Wang S, In K, Galano-Tan WC, Jun S, Cho K, Hwang YH, Kim SJ, Park W (2016) Reference values of hematology, biochemistry, and blood type in cynomolgus 
monkeys from cambodia origin. Lab Anim Res 32:46-55.

13. Kim HS, Byun D, Kim RG, Kang GH, Park JY, Yang YS, Han SC, Kim HI (2018) Simplified adaptor for stereotactic surgery in non-human primates. J Neurosci Methods 295:139-143.

14. Pellegrino LJ, Cushman AJ (1971) Use of stereotactic tech- nique. In: Methods in psychobiology (Meyers RD, ed), pp 6789. Academic Press, Cambridge, MA.

15. Hamlin H, Rakic P, Yakovlev PI (1965) Stereotactic imprecision. Confin Neurol 26:426-436. 